\title{
A Lightweight and Accurate Link Abstraction Model for the Simulation of LTE Networks in ns-3
}

\author{
Marco Mezzavilla ${ }^{\dagger}$, Marco Miozzo ${ }^{\S}$, Michele Rossi ${ }^{\dagger \ddagger}$, Nicola Baldo , Michele Zorzi ${ }^{\dagger \neq}$ \\ ${ }^{\dagger}$ Dept. of Information Engineering (DEI), Via Gradenigo 6B, 35131 Padova, Italy \\ ${ }^{\S}$ Centre Tecnológic de Telecomunicacions de Catalunya (CTTC), \\ Av. Carl Friedrich Gauss 7, 08860 Castelldefels, Barcelona, Spain \\ ${ }^{\ddagger}$ Consorzio Ferrara Ricerche (CFR), Via Saragat 1, 44122 Ferrara, Italy \\ \{mezzavil,rossi,zorzi\}@dei.unipd.it, \{mmiozzo,nbaldo\}@cttc.es
}

\begin{abstract}
In this work we present a link abstraction model for the simulation of downlink data transmission in LTE networks. The purpose of this model is to provide an accurate link performance metric at a low computational cost by relying solely on the knowledge of the SINR and of the modulation and coding scheme. To this aim, the model combines Mutual Information-based multi-carrier compression metrics with Link-Level performance curves matching, to obtain lookup tables that express the dependency of the Block Error Rate on the SINR values and on the modulation and coding scheme being used. In addition, we propose a 3GPP-compliant Channel Quality Indicator evaluation procedure, based on the proposed Link Abstraction Model, to be used as part of the LTE Adaptive Modulation and Coding mechanisms. Finally, we discuss how these contributions have been tested, validated and integrated in the ns-3 simulator. The link abstraction model described in this paper has been included in the official ns-3 distribution since release 3.14.
\end{abstract}

\section{Categories and Subject Descriptors}

I.6.8 [Simulation and Modeling]: Discrete event; I.6.5 [Model Development]: Modeling methodologies; C.2.1 [Computer Systems Organization]: Computer - Communication Networks - Network Architecture and Design - Wireless communication

\section{General Terms}

Algorithms, Design, Performance

\section{Keywords}

LTE; OFDM; ns-3; Link Abstraction; BLER; MIESM; AMC.

\section{INTRODUCTION}

Long Term Evolution (LTE) [21] is today's most advanced cellular network technology, and is expected to be massively deployed in the upcoming years. It is a very versatile system, featuring many possible configurations able to achieve different performance tradeoffs. In the perspective of deploying and managing future LTE net-

Permission to make digital or hard copies of all or part of this work for personal or classroom use is granted without fee provided that copies are not made or distributed for profit or commercial advantage and that copies bear this notice and the full citation on the first page. To copy otherwise, to republish, to post on servers or to redistribute to lists, requires prior specific permission and/or a fee.

MSWiM'12, October 21-25, 2012, Paphos, Cyprus.

Copyright 2012 ACM 978-1-4503-1628-6/12/10 ...\$15.00. works, operators and equipment vendors are strongly interested in identifying the configurations and solutions that can achieve the best possible performance in a variety of scenarios according to their needs. Ideally, this identification would be carried out experimentally by analyzing measurements obtained from an actually deployed network. However, doing so by either testbed experiments or field trials is a very time consuming and expensive process. Thus, simulation is an appealing alternative to perform some pre-tuning of the selected algorithms and protocols before they are deployed. In this respect, there is a tradeoff between choosing a very accurate simulation model, which typically has a high computational complexity and only allows for the simulation of a few network elements, and a more simplified model, which can scale to larger scenarios but which often has a limited accuracy.

The work that we present in this paper aims at reducing the gap between these two extremes. In detail, our aim is to provide an accurate and, at the same time, computationally lightweight Link Abstraction Model (LAM) for the LTE evolved Universal Terrestrial Radio Access (E-UTRA). This model shall allow the accurate prediction of transport block errors at the MAC layer taking into account channel fluctuations, multi-user interference as well as physical layer configurations (bandwidth assignment, modulation, coding, etc.). Thus, we integrate this model into the ns-3 NetworkLevel (NL) simulator of LTE, which accounts for multiple UEs and eNodeBs, and allows the simulation of a complete end-to-end LTE system, including architectural components. To this end, one might of course come up with a detailed implementation of the eUTRAN procedures [2] and especially of the LTE PHY (e.g., modeling its operations at the symbol level). However, this would lead to a very complex and computationally demanding model, which would not scale up to the medium and large network sizes typically considered for NL simulation. A more suitable approach, which is the one that we take here, is instead that of performing some offline preprocessing based on Link-Level (LL) simulations, so as to derive a simplified model of the influence of channel and system parameters on the PHY performance, represented by the Transport Block error rate. This pre-encoded mapping allows to retain a good amount of the accuracy of LL simulation when modeling phenomena such as multi-user interference, OFDMA bandwidth allocation and random channel realizations, while not retaining their complexity, thereby allowing for better scalability.

Evaluating the error distribution in OFDMA-based wireless systems is very challenging for a number of reasons. First of all, OFDM transmissions are typically used in scenarios affected by frequency selective fading, meaning that subcarriers may perceive very different channel gains. Besides, OFDMA further increases the system complexity as subcarriers are assigned to different users, whose signals are typically generated with different transmission 
powers and MCSs. This makes the task of predicting the error distribution per user rather complex, in terms of both collecting a reduced subset of parameters to describe performance trends, and generating a flexible error model in order to cover all possible scenarios. To address this problem, Link-to-System Mapping (LSM) has been previously proposed for use with generic multicarrier systems [11, 19,23]. The first practical application of the LSM approach to modeling a real wireless technology was the evaluation of the Worldwide Interoperability for Microwave Access (WiMAX) radio technology by the IEEE 802.16 task force; in this context, several LSM techniques were applied and evaluated [24]. On this matter, two extensions of the well known network simulator 2 (ns2) [5] called WINSE [8] and WiDe [17] had these solutions integrated; however, their code is not publicly available.

More recently, LSM has been extensively investigated for application to the LTE technology. Many papers have leveraged on LSM as part of simulation models aimed at the evaluation of interference management and allocation schemes $[9,16]$. However, only a few of them $[13,14]$ made the simulation tool publicly available. [13] refers to a set of Matlab simulators that aim at providing a comprehensive framework for the simulation of link and MAC layer performance. The design choices of Matlab and the focus on lower layer aspects do not give to this tool the possibility of evaluating complex network scenarios, featuring mobility and traffic constraints. Some of these assumptions have been relaxed in [14], where c++ was adopted as the programming language and some networking functionalities were included. However, this simulator is designed primarily to evaluate MAC-level performance and does not properly model the Evolved Packet Core (EPC), in charge of handling, among other aspects, bearers, their Quality of Service (QoS), and mobility.

Recently, a new module called LTE-EPC Network Simulator (LENA) [4] has been developed for LTE as an extension of the ns-3 simulator [6]. LENA already includes EPC functionalities [10] and is designed in a product oriented fashion (i.e., it implements the Scheduling APIs defined by the Small Cell Forum [22], formerly known as Femto Forum). It is to be noted that LENA has all the advantages of a large open source project, including the support of a lively community for what concerns debugging, validation and maintenance.

The main contribution of this work, which is discussed in the following sections, is the design and implementation of a lightweight link abstraction model for the downlink transmission of LTE systems, and its integration with LENA. In addition, we show how this model can be exploited to design an algorithm for reporting channel quality indicator (CQI) feedback according to the 3GPP guidelines in order to test the online selection of the Modulation and Coding Scheme (MCS) for each user, subject to given BLock Error Rate (BLER) requirements. As a further introductory remark, we would like to note that a version of LENA including the code of the model presented in this paper has been part of the official ns-3 distribution since release 3.14 .

\section{THE PROPOSED ERROR MODEL}

\subsection{Overview}

For an accurate evaluation of the user's performance, besides the MCS assigned by the LTE scheduler, it is important to track the residual errors, i.e., after link layer processing, that are due to channel phenomena such as fading, multiple-access interference, etc. However, as discussed above, a comprehensive simulation of link layer procedures would entail a high computational complexity, which is undesirable for multi-user scenarios. With BLER we refer to the residual error rate after all PHY layer procedures, i.e., affecting the code blocks at the output of the turbo decoder at the receiver side. The physical layer model of the LTE simulator returns SINR values $S I N R_{1}, S I N R_{2}, \ldots, S I N R_{N}$ for each Resource Block (RB) $n \in\{1,2, \ldots N\}$ for all users, calculated using an AWGN model and a Gaussian interference model. We recall that a RB corresponds to the allocation quantum in LTE, and is composed of 12 sub-carriers ( $15 \mathrm{kHz}$ each) and 14 OFDM symbols, transmitted over a Time Transmission Interval (TTI) of $1 \mathrm{~ms}$.

Following the Mutual Information Effective SINR Mapping (MIESM) method [12], for the transmission of each block we pick the instantaneous SINR vector $\left(S I N R_{1}, S I N R_{2}, \ldots, S I N R_{N}\right)$ and map it onto a Mean Mutual Information per coded Bit (MMIB) metric. The obtained MMIB is a time-varying compressed representation of the channel quality as perceived by any given user at any given time.

In addition, we store offline calculated curves returning the BLER as a function of the SINR for each valid (MCS, $\mathrm{CB}_{\text {size }}$ ) pair, where MCS is a modulation and coding scheme and $\mathrm{CB}_{\text {size }}$ represents the code block size. These curves have been obtained with the Vienna link level simulator $[7,18]$.

Finally, this offline calculated SINR to BLER mapping is utilized, together with the instantaneous MMIB information, to obtain the BLER traces for each user. This procedure is explained in greater detail in the following Section 2.2.

\subsection{Effective SINR Mapping model}

The link level simulations executed to build our abstraction model assume a frequency flat channel response at any given SINR (the so-called AWGN channel). Let us consider a given LTE user and let $S I N R_{n}$ be the instantaneous SINR value associated with RB $n$, where $n=1,2, \ldots, N$ and $N$ is the number of RBs allotted to this user.

Given this, let us assume that the simulator returns an instantaneous SINR sample for each RB, which means a vector $\left(S I N R_{1}\right.$, $\left.S I N R_{2}, \ldots, S I N R_{N}\right)$. In order to obtain a lightweight and effective mapping from this vector to a single BLER metric we consider the effective SINR mapping (ESM) method, see [3]. Briefly, the instantaneous SINR vector is mapped onto a single scalar value as follows [19]:

$$
e S I N R=\alpha_{1} I^{-1}\left(\frac{1}{N} \sum_{n=1}^{N} I\left(\frac{S I N R_{n}}{\alpha_{2}}\right)\right)
$$

where $I(\cdot)$ represents the information measure function, $I^{-1}(\cdot)$ is its inverse, whereas $\alpha_{1}$ and $\alpha_{2}$ are two scaling parameters that are tuned as a function of the selected MCS. These parameters are specified in Section 2.3 along with function $I(\cdot)$.

The results provided in [12] demonstrate that the MIESM method outperforms all the other mapping approaches in terms of approximation accuracy for the BLER curves. Thus, we adopted the Mutual Information (MI) metric for our implementation.

\subsection{MIB Mapping}

The MIB is defined in [3] as the mutual information between the bit input belonging to a specific constellation (MCS), and the corresponding log-likelihood ratio (LLR) output at the receiver.

As reported in [3], it can be approximated through the following 
function: ${ }^{1}$

$$
J(t)=\left\{\begin{array}{ll}
0, & t<0.001 \\
a_{1} t^{3}+b_{1} t^{2}+c_{1} t, & 0.001 \leq t<1.6363 \\
1-e^{\left(a_{2} t^{3}+b_{2} t^{2}+c_{2} t+d_{2}\right)}, & 1.6363 \leq t \leq 50 \\
1, & t>50
\end{array},\right.
$$

where the parameters have been obtained through numerical fitting and are reported in the following Table 1.

\begin{tabular}{|l|l|}
\hline$a_{1}=-0.04210661$ & $a_{2}=0.00181492$ \\
\hline$b_{1}=0.209252$ & $b_{2}=-0.142675$ \\
\hline$c_{1}=-0.00640081$ & $c_{2}=-0.0822054$ \\
\hline- & $d_{2}=0.0549608$ \\
\hline
\end{tabular}

Table 1: J-function approximation parameters

Specifically, it has been demonstrated [3] that the MIB of any modulation $m$ can be approximated as a mixture of $J(\cdot)$ functions as follows:

$$
I_{m}(x) \simeq \sum_{k=1}^{K} \alpha_{k} J\left(\beta_{k} \sqrt{x}\right)
$$

where $\sum_{k=1}^{K} \alpha_{k}=1$ for some $K \geq 1$ and the argument $x$ is the SINR associated with the transmission channel under study. Numerical fittings have been carried out (see again [3]) to obtain $K$, $\alpha_{k}$ and $\beta_{k}$ for QPSK, 16-QAM and 64-QAM, as reported in the following Table 2 .

\begin{tabular}{|c|c|}
\hline Modulation $m$ & MIB function $I_{m}(x)$ \\
\hline \hline QPSK & $J(2 \sqrt{x})$ \\
\hline 16-QAM & $\frac{1}{2} J(0.8 \sqrt{x})+\frac{1}{4} J(2.17 \sqrt{x})+\frac{1}{4} J(0.965 \sqrt{x})$ \\
\hline 64-QAM & $\frac{1}{3} J(1.47 \sqrt{x})+\frac{1}{3} J(0.529 \sqrt{x})+\frac{1}{3} J(0.366 \sqrt{x})$ \\
\hline
\end{tabular}

Table 2: Numerical approximations for MIB mapping

As above, with $S I N R_{n}$ we mean the instantaneous SINR value associated with RB $n$, where $n=1,2, \ldots, N$ and $N$ is the number of RBs allotted to the user. According to the above discussion, the function $I_{m}(x)$ can be used to map $S I N R_{n}$ onto the corresponding mutual information domain, where $m$ is the adopted modulation scheme. Note that the argument $x$ corresponds to $S I N R_{n}$ and in LTE, for each sub-frame, the same modulation is picked for all RBs. Given all that, the Mean Mutual Information per coded Bit (MMIB) can be obtained as follows:

$$
M M I B=\frac{1}{N} \sum_{n=1}^{N} I_{m}\left(S I N R_{n}\right),
$$

where $N$ is the number of RBs assigned to a specific user and $m$ is the modulation that this user is exploiting. To sum up, the model starts by evaluating the mutual information value for each RB from the corresponding SINR samples. Subsequently, the MMIB is computed by averaging (effective SINR mapping) the corresponding mutual information values as per Eq. (4).

\subsection{BLER prediction}

The data at the MAC layer of the LTE protocol stack (right above the LTE PHY) is arranged in Transport Blocks (TB), whose size depends on the specific configuration of the underlying PHY. TBs are

${ }^{1}$ Note that, compared to [3], we have truncated the function to avoid that it takes values outside the $[0,1]$ interval.

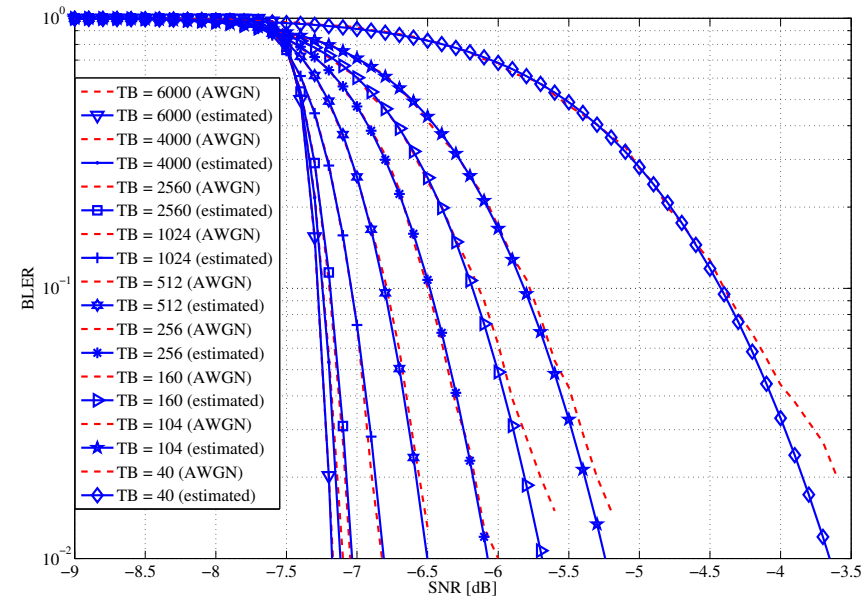

Figure 1: BLER vs SNR for MCS 1

split into a number of CBs which are independently encoded by the turbo encoder at the PHY layer. Each CB is then encoded and transmitted over the channel exploiting the $N$ RBs allotted to the user. In this section we show how to efficiently compute the Transport BLock Error Rate (TBLER) from the results of Section 2.3.

For the moment, let us focus on the $i$-th CB of a given TB. As mentioned in Section 2.1, link-level simulations (whose results were obtained using the Vienna LL simulator) have been used to obtain the PHY layer performance in terms of BLER vs SINR over AWGN channels, accounting for the configuration of the PHY layer turbo encoder in terms of Code Block (CB) length and selected MCS. The 3GPP standard has been considered to assess the correct $\mathrm{CB}$ sizes in the simulations, according to [2]. As an example, the dotted lines in Fig. 1 show the BLER as a function of SINR for MCS 1. These curves have been calculated offline considering the LTE PHY layer procedures implemented in the LTE Downlink LL Vienna Simulator [7], as described in [15].

As can be seen from these plots, the CB size highly impacts the actual BLER performance for a given MCS.

As mentioned above, the selected CB $i$ is transmitted over the channel using the $N$ RBs that are assigned to the user. At the receiver side, a reference SINR value ${ }^{2}$ is made available by the PHY layer model of the ns-3 simulator for each of these RBs, returning the SINR vector $\left(S I N R_{1}, S I N R_{2}, \ldots, S I N R_{N}\right)$, as discussed in Section 2.1. From here, we obtain the MMIB metric using Eq. (4), as explained in Section 2.3. This MMIB corresponds to an equivalent SINR for the transmission of CB $i$ over the allotted $\mathrm{RBs}$. As a last step to obtain the residual error rate of $\mathrm{CB} i$, we need to map its MMIB onto the corresponding BLER, which is referred to here as $C B L E R_{i}$. This is done according to the following procedure.

In order to reduce the computational burden at simulation time as much as possible, an approximation based on the Gaussian cumulative model has been adopted. According to this, the estimated BLER curves as a function of MMIB are parameterized as follows:

$$
C B L E R_{i}(x)=\frac{1}{2}\left[1-\operatorname{erf}\left(\frac{x-b_{S, M}}{\sqrt{2} c_{S, M}}\right)\right],
$$

where $b_{S, M}$ and $c_{S, M}$ are the mean and the standard deviation

${ }^{2} \mathrm{We}$ assume no frequency selectivity among the 12 sub-carriers composing the resource block. 
of the Gaussian cumulative distribution, respectively, and $x$ is the MMIB associated with CB $i$. $S$ is the code block size and $M$ is the MCS, which dictates the actual transmission rate. What we did at this point, was to find suitable pairs $\left(b_{S, M}, c_{S, M}\right)$ for each MCS and code size. We did so through numerical fitting so that the curves from Eq. (5) would match those obtained from the Vienna LL simulator. The result of this procedure is shown in Fig. 1, where the solid curves represent the result of Eq. (5) where we have used the best fitting $\left(b_{S, M}, c_{S, M}\right)$ pair for each MCS and code size. As can be seen from these plots, the approximated BLER from Eq. (5) (solid lines) closely match the BLER obtained through the numerical simulation of LTE PHY procedures (dotted lines). The overall Transport BLock Error Rate (TBLER) is thus found as:

$$
T B L E R=1-\prod_{i=1}^{C}\left(1-C B L E R_{i}\right),
$$

where $C$ is the number of CBs contained in the TB.

Lookup tables: to limit the computational complexity and the memory space taken by the proposed link abstraction model, we only considered a subset of CB sizes, i.e., $\mathcal{S}=\{40,104,160,256$, $512,1024,2560,4000,6000\}$ bits. This choice is aligned with the typical performance of turbo codes, where large CB sizes do not strongly affect BLER performance. However, we note that for CB sizes smaller than 1000 bits, the BLER performance might significantly differ as we vary the block size (up to nearly $3 \mathrm{~dB}$ ). Therefore, we accounted for an unbalanced quantization of $\mathrm{CB}$ sizes in order to get more accuracy in the critical zone (small code blocks). This is particularly evident from Fig. 1 that shows a similar BLER profile for large CB sizes (e.g., 2500, 4000 and 6000 bits), whereas the performance gap increases as the $\mathrm{CB}$ size gets smaller. Thus, $\left(b_{S, M}, c_{S, M}\right)$ parameters have been tabulated for all valid combinations of MCS and block sizes in set $\mathcal{S}$. We remark that high MCS values with high order modulations and efficient coding rate schemes, such as 64-QAM with an Effective Coding Rate (ECR) of 0.92 (i.e., MCS 29), allow for a minimum CB size of 2560 bits. The latter is much larger than the minimum size at small MCS values, e.g., MCS 1, where the minimum size is 40 bits, see Fig. 1. This reflects the fact that turbo coding offers better performance as the code block size increases; thus, for high order modulations such as MCS 29, small code block lengths are inefficient as the resulting BLER performance is unacceptable.

\section{LINK ADAPTATION IMPROVEMENT}

In this section we propose an improved MCS assignment scheme supported by a new CQI evaluation scheme based on 3GPP guidelines. Note that this novel algorithm for CQI evaluation could not be tested in the previous ns-3 distribution as it is based on residual error estimates.

Link adaptation plays a fundamental role in modern wireless communications systems, which need to face issues such as strong interference from multiple users and their mobility, which makes the wireless channel frequency selective. These facts are coped with by LTE adaptive modulation and coding algorithms. Focusing on the downlink scenario, AMC has the role of tracking the perceived SINR and sending back to the base station (eNodeB) a so called CQI report. Hence, periodically, the UE reports to the eNodeB a single CQI value for all the RBs (the so called wideband CQI). This information is a "compressed" representation of the quality experienced by the UE in a specific sub-frame and is used at the base station side for the selection of the MCS. This process is continuously executed so as to adapt to channel and network dynamics.
Our proposed MCS assignment scheme relies on an SINR to CQI mapping approach based on the link error abstraction model presented in the previous section. As a competing approach we consider the algorithm that is currently implemented in the LENA ns-3 simulator, which is inspired by the spectral efficiency concept, see also [20].

Spectral efficiency-based approach: consider the generic RB $n$, and let $S I N R_{n}$ be the corresponding SINR value, in linear units. We obtain the spectral efficiency $\eta_{n}$ of RB $n$ using the following equations:

$$
\begin{gathered}
\Gamma=-\frac{\ln (5 \mathrm{BER})}{1.5}, \\
\eta_{n}=\log _{2}\left(1+\frac{S I N R_{n}}{\Gamma}\right),
\end{gathered}
$$

where BER is the Bit Error Rate and $\Gamma$ is the so called SNR gap, as it models the discrepancy between practical implementations and information-theoretic results.

Upon the calculation of $\eta_{n}$, which lies in the continuous interval $[0.15,5.55]$, the procedure described in [1] is used to derive the corresponding CQI, which is a quantized version of $\eta_{n}$.

Error model-based approach: this model relies on the exploitation of our link abstraction model. Thanks to this approach, we can dynamically select the MCS that better complies with a given target transport block error rate for the connection, referred to as $T B L E R_{\mathrm{th}}$. In the following, we describe our improved CQI evaluation procedure by abstracting away from the actual implementation details, i.e., on the actual representation of CQI values (at the receiver, e.g., number of CQI levels, etc.) and the subsequent mapping of these CQIs onto a suitable MCS (which is done at the eNodeB).

Our procedure works as follows. Periodically, each UE computes its received power spectrum profile, i.e., an SINR sample is acquired for each possible RB. ${ }^{3}$ For any user $i=1,2, \ldots, N_{U E}$, it starts from MCS 29, which corresponds to the most aggressive transmission scheme, and evaluates the TBLER performance considering a transport block composed of all possible LTE RBs. The transport block error rate for user $i, T B L E R_{i}$, is estimated through Eq. (5), taking as input the vector of SINRs for the selected user, and the MCS that we are currently evaluating. If $T B L E R_{i}$ is larger than or equal to the target BLER defined by 3GPP (i.e., 0.1 ) [2], we keep on searching for a better (less aggressive) MCS; otherwise, the procedure stops. Thus, the corresponding CQI is obtained in order to satisfy the spectral efficiency constraints defined by the standard [2].

\section{SIMULATION RESULTS}

In the following we provide some technical results for selected LTE scenarios. Our main goals are: 1) to illustrate the usability of the proposed link abstraction model, and 2) to prove the efficiency of the link adaptation improvement proposed in Section 3. We would like to note that the validation of the error model proposed is simplified for the sake of readability; a more in depth validation can be found in the LENA documentation [4]. In Table 3 we report the considered system parameters.

First of all we would like to present some quantitative results on the computational complexity of the model. The simulator presented in this paper takes 23 seconds for simulating a 10 seconds scenario with one UE transmitting continuously at full bandwidth

${ }^{3}$ In this case, all RBs allowed by the selected LTE channel bandwidth are accounted for. 


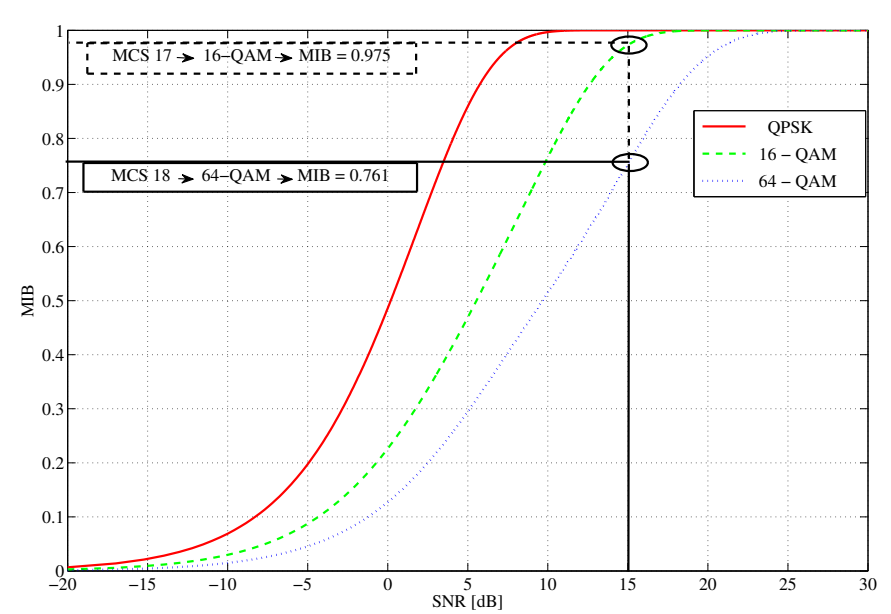

Figure 2: MI extraction

\begin{tabular}{|c|c|}
\hline \multicolumn{2}{|c|}{ PHY Configuration } \\
\hline \hline Parameter & Value \\
\hline Frequency & $2.1 \mathrm{GHz}$ \\
\hline Channel Bandwidth & $5 \mathrm{MHz}$ \\
\hline Propagation Model & Friis free-space \\
\hline Number of $R B s$ & 25 \\
\hline$R B_{\text {bandwidth }}$ & $180 \mathrm{kHz}$ \\
\hline$R B_{\text {subcarriers }}$ & 12 \\
\hline$R B_{\text {OF DMsymbols }}$ & 14 \\
\hline eNodeB TX power & $30 \mathrm{dBm}$ \\
\hline Noise spectral density $\left(\mathrm{N}_{0}\right)$ & $-174 \mathrm{dBm} / \mathrm{Hz}$ \\
\hline
\end{tabular}

Table 3: Main system parameters

to an eNB, while the same scenario takes 38 minutes when simulated with the LTE Vienna Link Layer simulator. ${ }^{4}$

1) Error model: we consider a downlink transmission from an eNodeB to a single static UE. For the wireless channel, we account for a Friis free-space propagation model, but note that the conclusions that we draw here are general and apply to more sophisticated models as well. The UE is placed 2150 meters away from the eNodeB and, according to the considered propagation loss model, it experiences an SINR of $15.1 \mathrm{~dB}$ for all its RBs.

We first evaluate the BLER performance resulting from the selection of a "safe" transmission scheme, MCS 17, which corresponds to 16-QAM (4 bits per OFDM symbol). As we now show, for this MCS the estimated error rate (through Eq. (5)) is below the standard TBLER threshold of $T B L E R_{\mathrm{th}}=0.1$. First of all, we extract the mutual information value associated with the experienced SNR, as shown in Fig. 2 (where we plot the approximation functions of Table 2). According to [2], from the selected MCS and the maximum number of assignable RBs, the TB size is 7272 bits (including the header). Following [2], the TB is split into two code blocks, $C B_{1}$ and $C B_{2}$, of size 3684 and 3584 bits, respectively. As shown in Fig. 3, these code sizes are mapped onto the closest CB size in set $\mathcal{S}$. In fact, as per our discussion in Section 2.4, in our ns-3 simulator fitting parameters are only stored for a subset of all possible CB sizes. Thus, the resulting CB size that will be used for the prediction of the CBLER performance is 2560 bits. Now,

\footnotetext{
${ }^{4}$ The reference hardware platform is an Intel Core2 Quad CPU Q8400 2.66GHz.
}

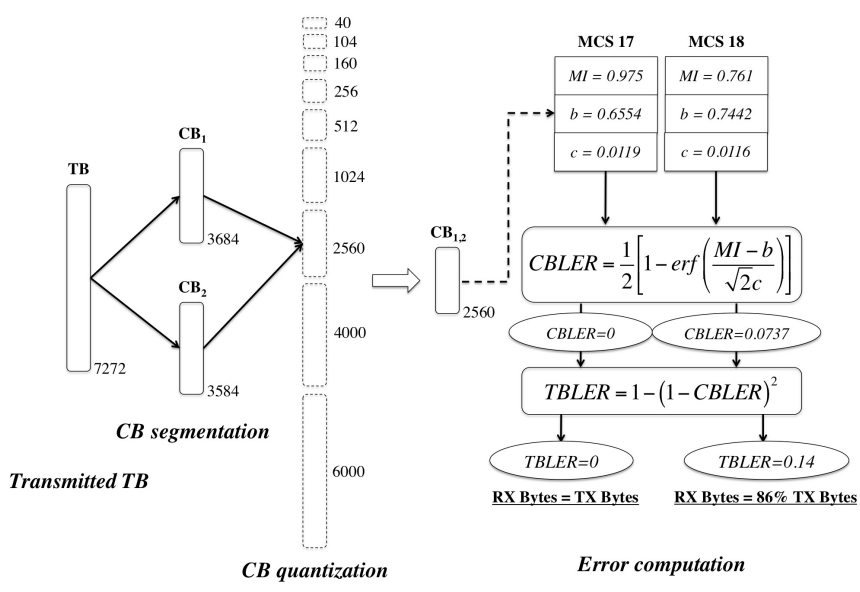

Figure 3: TBLER computation

using the $b_{S, M}$ and $c_{S, M}$ parameters associated with the latter code block size and the previously extracted MI with Eqs. (5) and (6), we obtain an estimated transport block error rate of $T B L E R=0$.

Next, we try to allocate a more aggressive modulation and coding scheme, MCS 18, for which the modulation order amounts to 6 bits per OFDM symbol (64-QAM). Thus, we repeat the procedure illustrated in the previous paragraph, obtaining the mutual information MI, and the fitting parameters $b_{S, M}$ and $c_{S, M}$. These quantities, together with Eqs. (5) and (6), return TBLER $=0.14$, which means that MCS 18 is not compatible with the considered error requirements.

2) Link adaptation: we now consider a scenario with a single UE, and we vary its distance from the eNodeB. This leads to SNR values ranging from about 2 to $30 \mathrm{~dB}$. Also, we consider the standard target transport block error rate of $T B L E R=0.1$. In Fig. 4, we show the effective spectral efficiency as a function of the SNR for the spectral efficiency-based (SE_MCS) and the errorbased (EM_MCS) MCS selection schemes. The effective spectral efficiency metric reflects the actual bits per second per unit of frequency that are successfully transmitted from the eNodeB to the UE, by also accounting for the residual error after PHY layer processing. As can be seen from Fig. 4, EM_MCS outperforms the current approach, at all SNR levels. This indicates that SE_MCS tends to be too conservative, even though a more aggressive technique can be used while still adhering to the target error requirements.

\section{CONCLUSIONS \& FUTURE WORK}

In this work we introduced a link abstraction model for the simulation of Transport Block errors in the downlink of an LTE system. Our objective here has been to provide a lightweight but still accurate procedure for the computation of the residual errors, after PHY layer processing, without having to go through the detailed simulation of LTE PHY procedures. Toward this end, we combined Mutual Information-based multi-carrier compression metrics with Link-Level performance curves matching. This allowed us to obtain pre-calculated lookup tables, which can be used in an online fashion to track residual bit errors after physical layer modulation and coding procedures. In addition, we have proposed a CQI evaluation procedure which can be used as part of the LTE Adaptive Modulation and Coding scheme, showing its superiority in terms of achievable spectral efficiency with respect to current ns-3 solutions. An implementation of both the link abstraction model and 


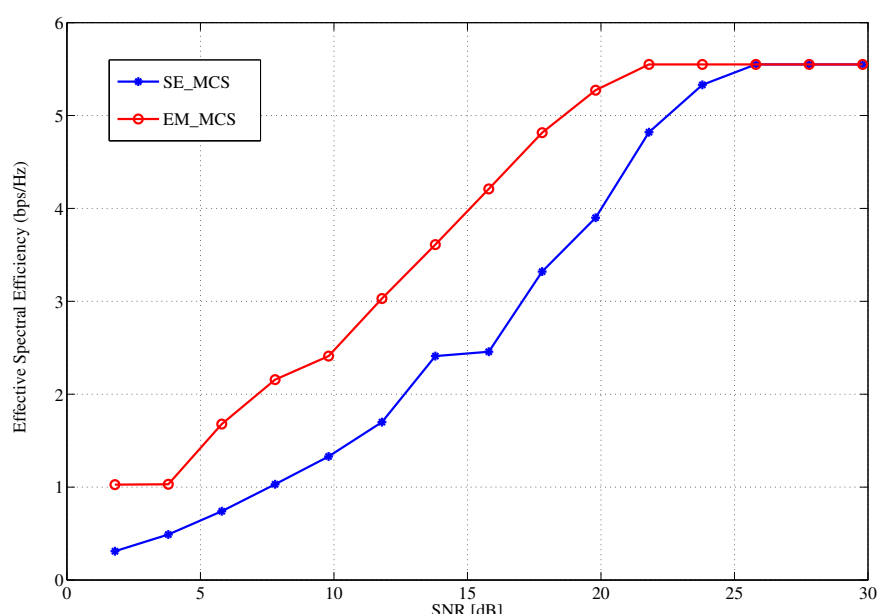

Figure 4: MCS assignment comparison

the CQI evaluation procedure is included with the official distribution of the ns-3 simulator since release 3.14, which is open source and publicly available. As future work we plan to investigate algorithms for resource allocation, especially targeting the transmission of streaming flows over LTE networks.

\section{ACKNOWLEDGMENTS}

The research leading to these results has received funding from the European Community Seventh Framework Programme (FP7ICT-2009-5) under grant agreement n. 258053 (MEDIEVAL project). The work at CTTC was partially supported by Ubiquisys as part of the LENA project, by the Spanish Ministry of Science and Innovation under grant number TEC2011- 29700-C02-01 (project SYMBIOSIS), and by the Catalan Regional Government under grant 2009SGR-940.

\section{REFERENCES}

[1] 3GPP R1-081483, "Conveying MCS and TB size via PDCCH".

[2] 3GPP TS 36.213, "E-UTRA Physical layer procedures".

[3] IEEE 802.16 Broadband Wireless Access Working Group, Evaluation Methodology Document (EMD).

[4] The LTE-EPC Network Simulator (LENA) project, http://iptechwiki.cttc.es/LTEEPC_Network_Simulator_(LENA).

[5] The Network Simulator - ns-2, http://www.isi.edu/nsnam/ns/.

[6] The Network Simulator - ns-3, http://www.nsnam.org/.

[7] The Vienna LTE Simulators, http://www.nt.tuwien.ac.at/about-us/staff/josep-colomikuno/lte-simulators/.

[8] A. Sayenko, O. Alanen, H. Martikainen, V. Tykhomyrov and O. Puchko. WINSE: WiMAX NS-2 Extension. In ICST Simulation Tools and Techniques (SIMUTools), March 2009.

[9] B. Sadiq, R. Madan and A. Sampath. Downlink Scheduling for Multiclass Traffic in LTE. In EURASIP Journal on Wireless Communications and Networking, March 2009.

[10] N. Baldo, M. Requena-Esteso, J. Nin-Guerrero, and M. Miozzo. A new model for the simulation of the LTE-EPC data plane. In ICST Workshop on ns-3 (WNS3), March 2012.

[11] Y. Blankenship, P. Sartori, B. Classon, V. Desai, and K. Baum. Link error prediction methods for multicarrier systems. In IEEE Vehicular Technology Conference (VTC), September 2004

[12] K. Brueninghaus, D. Astely, T. Salzer, S. Visuri, A. Alexiou, S. Karger, and G.-A. Seraji. Link performance models for system level simulations of broadband radio access systems. In IEEE Personal, Indoor and Mobile Radio Communications (PIMRC), September 2005.

[13] C. Mehlfuhrer, J. Colom Ikuno, M. Simko, S. Schwarz, M. Wrulich and M. Rupp. The Vienna LTE simulators: Enabling reproducibility in wireless communications research. In EURASIP Journal on Advances in Signal Processing, July 2011.

[14] G. Piro, L.A. Grieco, G. Boggia, F. Capozzi and P. Camarda. Simulating LTE Cellular Systems: An Open-Source Framework. In IEEE Transactions on Vehicular Technology, pages 498-513, February 2011.

[15] J. Colom Ikuno, M. Wrulich and M. Rupp. Performance and Modeling of LTE-HARQ. In ITG Workshop on Smart Antennas (WSA), February 2009.

[16] J. Fan, Q. Yin, G.Y. Li, B. Peng and X. Zhu. MCS Selection for Throughput Improvement in Downlink LTE Systems. In International Conference on Computer Communications and Networks (ICCCN), August 2011.

[17] M. Miozzo and F. Bader. Accurate Modelling of OFDMA Transmission Technique using IEEE $802.16 \mathrm{~m}$ Recommendations for WiMAX Network Simulator Design. In ICST Conference on Mobile Networks and Management (MONAMI), September 2009.

[18] C. Mehlfuhrer, M. Wrulich, J. C. Ikuno, D. Bosanska, and M. Rupp. Simulating the Long Term Evolution Physical Layer. In European Signal Processing Conference (EUSIPCO 2009), August 2009.

[19] M. Moisio and A. Oborina. Comparison of Effective SINR Mapping with Traditional AVI Approach for Modeling Packet Error Rate in Multi-State Channel. In Next Generation Teletraffic and Wired/Wireless Advanced Networking (NEW2AN), June 2006.

[20] H. Seo and B. G. Lee. A proportional-fair power allocation scheme for fair and efficient multiuser OFDM systems. In IEEE Global Telecommunications (GLOBECOM), December 2004.

[21] S. Sesia, I. Toufik, and M. Baker. LTE: The UMTS Long Term Evolution - from Theory to Practice. John Wiley \& Sons, second edition, August 2011.

[22] SmallCell-Forum. LTE MAC Scheduler Interface Specification v1.11, October 2010.

[23] E. Tuomaala and H. Wang. Effective SINR approach of link to system mapping in OFDM/multi-carrier mobile network. In Mobile Technology, Applications and Systems, November 2005.

[24] WiMAX Forum. WiMAX System Evaluation Methodology Document. Version 1.0, January 2007. 